\title{
LOS EFECTOS DEL INVOLUCRAMIENTO PARENTAL SOBRE EL DESEMPEÑO ACADÉMICO DE ESTUDIANTES EN UN GRUPO DE ESCUELAS PARTICULARES DE PUERTO PRÍNCIPE, HAITÍ
}

\section{THE EFFECTS OF PARENTAL INVOLVEMENT ON THE ACADEMIC PERFORMANCE OF STUDENTS IN A GROUP OF PRIVATE SCHOOLS OF PORT-AU-PRINCE, HAITI}

\author{
Ganddey Milorme \\ Université Adventiste d'Haïti \\ ganddeym@yahoo.fr \\ https://orcid.org/0000-0003-4164-1982
}

\begin{abstract}
RESUMEN
La relación entre la participación de los padres en la vida escolar de sus hijos y el rendimiento académico ha sido objeto de muchos estudios. Sin embargo, muy pocos han investigado esa relación en las escuelas de Puerto Príncipe, Haití. Esta investigación de tipo cuantitativo, descriptivo y correlacional tuvo como objetivo identificar la relación entre el grado de involucramiento parental y el rendimiento académico de 128 estudiantes del noveno año de un grupo de escuelas particulares de Puerto Príncipe durante el año académico 2016-2017. Los resultados del estudio muestran que la mayoría de las dimensiones de la participación de los padres, formuladas por Epstein, son predictoras del rendimiento académico de los estudiantes. También se encontró que el aprendizaje en el hogar es el mejor indicador del rendimiento académico entre los participantes y que el rendimiento académico más alto de los estudiantes se observó en el curso de Criollo, seguido de Ciencias sociales y de Francés. La correlación positiva más fuerte con el involucramiento parental se observó en las Ciencias Sociales. Se concluye que la participación de los padres en la vida escolar es un predictor del rendimiento académico.
\end{abstract}

Palabras clave: involucramiento parental, rendimiento académico

\section{ABSTRACT}

The relationship between parents 'participation in their children's school life and academic performance has been the subject of many researches. However, very few have studied this relationship in schools in Port-au-Prince, Haiti. This research of a quantitative, descriptive and correlational type, aimed to identify the relationship between the degree of parental involvement and the academic performance of the 128 ninth-grade students from a group of private schools in Port-au-Prince during the academic year 20162017. The study results show that most dimensions of parental involvement, 


\section{MILORME}

formulated by Epstein, are predictors of student academic achievement. It was also found that learning at home is the best indicator of academic performance in the ninth grade, and the highest academic performance of the students was observed in the Creole course, followed by the Social Sciences and French. The strongest positive correlation with parental involvement was observed in Social Sciences. It is concluded that the participation of parents in school life is a predictor of academic performance.

Keywords: parental involvement, academic performance

\section{Introducción}

La familia es el primer y principal sistema social en el que los niños pequeños comienzan a adquirir las habilidades cognitivas y sociales necesarias para la escuela. Los padres pueden desempeñar un papel vital en el aprendizaje y desarrollo de sus hijos involucrándose como socios de aprendizaje durante los años escolares hasta la edad adulta temprana.

En los países pobres, como algunos países de África, el problema del fracaso escolar excede los límites razonables, ya que afecta a la gran mayoría de los estudiantes. En el año 2008, el Banco Mundial y la Unesco estimaron que la tasa de finalización de la escuela primaria era del 39,7\% en la República Centroafricana y del 65\% en Malawi (Banco Mundial, 2009). Por otro lado, el análisis del sistema educativo de Haití, producido en el Plan Operativo 2010-2015 por el Ministerio de Educación Nacional y Formación Profesional (MENFP, 2012), reveló una tasa de eficiencia terminal de entre el 40 y el 50\%. Estos datos permiten comprender que el fracaso escolar es un problema persistente que no sólo afecta a la educación de los países pobres sino, en cierta medida, a todos los sistemas educativos.

El Ministerio de Educación Nacional y Formación Profesional (MENFP, 2012) admite que "el sistema educativo se enfrenta a problemas tanto en térmi- nos de oferta (acceso y participación), la eficiencia interna, como en la calidad, la eficiencia externa y gobernanza" (p. 39). Los datos muestran que sólo el 19\% de los niños que comienzan la escuela a la edad de 6 años completan al menos un año de la escuela secundaria. Y, de los que entran en séptimo año de educación básica, sólo el 7,83\% logran obtener el diploma de fin de estudios clásicos.

Según los análisis del MENFP, este bajo rendimiento académico registrado en el sistema se debe, entre otras razones, a las malas condiciones de la enseñanza y el aprendizaje, que resulta de una combinación de factores, incluyendo la retirada de los principales actores de la vida escolar, como los padres y profesores.

Diversos estudios (Bergeron, Rousseau y Leclerc, 2011; Blain 1992; Foucault, 2017; Mestari, 2001) han confirmado que todos los niños pueden tener éxito e, incluso, han propuesto formas de mejorar las prácticas actuales. Convencidos de esta premisa, muchos países como Francia, Estados Unidos y más tímidamente Haití con la reforma de Bernard, han puesto en marcha iniciativas para movilizar a todas las partes interesadas en el sistema educativo, en particular a maestros y padres, en torno de acciones adaptadas de enseñanza y supervisión que puedan garantizar el éxito de todos los niños sin distinción. 
Con una buena sinergia de acción entre todos los actores, no hay razón para perder un solo cerebro.

De lo anterior sobre el fracaso escolar, es importante preguntar:

1. ¿Por qué en una cohorte de estudiantes que tienen las mismas oportunidades de aprendizaje, algunos tienen éxito y otros no?

2. ¿Qué diferencia puede hacer la participación de los padres para mejorar el rendimiento académico de los estudiantes?

3. ¿Qué dimensión de participación de los padres influye más en el rendimiento académico de los estudiantes de noveno grado?

La respuesta a estas preguntas requiere una investigación a fondo. Por lo tanto, esta investigación buscó explorar y profundizar en los efectos de la participación de los padres en la vida escolar y el rendimiento académico de los estudiantes. Los resultados pueden ayudar a fortalecer la sinergia entre los padres y la escuela, para un mejor rendimiento académico de los estudiantes y un mejor gobierno de las escuelas en Haití.

\section{Los padres, ayudantes del profesor}

Desde hace varios años, el tema de la participación de los padres en la vida escolar siempre ha atraído la atención de los investigadores. Hoy se reconoce que los padres son colaboradores esenciales de los maestros. Duval, Dumoulin y Perron (2014) sugieren que los hábitos de la vida de los padres (puntualidad, hora de dormir, ocio, comida, etc.), así como su apoyo al trabajo escolar en el hogar y el acompañamiento para resolver problemas de conducta o administrativos, ayudan a prevenir el abandono escolar y a mejorar el rendimiento de los niños.

\section{Los investigadores y la participación de los padres}

Muchos investigadores están dedicados a identificar los vínculos entre la participación de los padres y el rendimiento de los estudiantes. La investigación de Epstein sobre este tema ha guiado las reflexiones de varios investigadores, como Benner, Boyle y Sadler (2016) y Castro et al. (2015). Epstein (citado en Doucet, Utzschneider y Bourque, 2009) sostiene que el apoyo de los padres en su papel como educadores es citado a menudo por los investigadores como medida educativa probable para contrarrestar las dificultades $y$, por lo tanto, para promover el éxito académico. Una relación positiva entre el involucramiento parental y el rendimiento académico ha sido establecida por muchos investigadores, incluyendo a Alghazo y Alghazo (2015) y Oyerinde (2014).

\section{Modo de participación de los padres}

Algunos profesionales de la educación son críticos de la participación de los padres en la vida escolar. Sin embargo, numerosos estudios (Flores de Apodaca, Gentling, Steinhaus y Rosenberg, 2015; Grolnick, 2016; Nanhou, Desrosiers y Belleau, 2013) revelan los aspectos positivos del involucramiento de los padres en la educación de sus hijos. En Haití, donde la oferta de la educación privada es alta, según el MENFP (2012), casi el 90\% de la financiación de los servicios educativos sigue siendo plenamente la responsabilidad de las familias.

Desde la perspectiva de varios investigadores (Alghazo y Alghazo, 2015; Castro et al., 2015; Flores de Apodaca, Gentling, Steinhaus y Rosenberg, 2015; Ross, 2016), los padres 
pueden participar de diferentes maneras en la vida escolar de sus hijos. Por ejemplo, pueden animarlos, ayudarlos con la tarea, hablar con ellos sobre su progreso y sus debilidades, ser voluntarios en la escuela, participar en comités y reuniones de padres, etc. Cada una de las formas de participación influye en el rendimiento académico de los estudiantes. A partir de estos tipos de participación de los padres, Castro et al. (2015) observaron que el tiempo dedicado a la tarea y a otras actividades de aprendizaje en los niños es una de las variables más fuertemente ligadas a un alto rendimiento académico.

También hay un fuerte vínculo entre el rendimiento académico y las altas aspiraciones de los padres. Cuanto más altas son las aspiraciones académicas de los padres, más se involucran en las actividades de aprendizaje de los niños y mayor es el rendimiento académico de los mismos.

\section{La importancia de la participación de los padres}

La cooperación efectiva entre la familia y la escuela es considerada uno de los elementos más importantes para prevenir el abandono y fomentar el éxito académico. Es evidente que cuando los maestros y los padres están unidos, no solo establecen una de las asociacio- nes más poderosas que requiere un niño para promover el éxito en la escuela, sino que también crean un ambiente seguro que fomenta el desarrollo completo del niño.

La relación entre la participación de los padres y el rendimiento académico ha sido objeto de muchos estudios a través del tiempo y el espacio (Alhosani, Singh y Al Nahyan, 2017, Thomas, De Backer, Peeters y Lombaerts, 2019; Wilder, 2014). Sin embargo, muy pocos estudios se han centrado en las escuelas particulares de Haití. Para llenar este vacío, se llevó a cabo este estudio de los efectos del involucramiento parental sobre el rendimiento académico de los estudiantes del noveno grado de un grupo de escuelas particulares del área metropolitana de Puerto Príncipe, Haití.

\section{Metodología \\ Tipo de investigación}

Esta investigación utilizó un abordaje cuantitativo, descriptivo y correlacional.

\section{Participantes}

La muestra estuvo conformada por padres y estudiantes de noveno año en cuatro escuelas primarias particulares, situadas en la zona metropolitana de Puerto Príncipe, Haití, como puede verse en la Tabla 1.

\section{Tabla 1}

Distribución de los estudiantes por escuela y número de cuestionarios

\begin{tabular}{lccccc}
\hline & \multicolumn{5}{c}{ Cuestionarios } \\
\cline { 2 - 6 } \multicolumn{1}{c}{ Escuela } & Iniciales & Abandono & Cancelados & No recogidos & Disponibles \\
\hline Escuela A & 26 & 4 & 2 & 1 & 19 \\
Escuela B & 59 & 10 & 1 & 2 & 46 \\
Escuela C & 9 & 2 & & 0 & 7 \\
Escuela D & 70 & 6 & 6 & 2 & 56 \\
Total & 164 & 22 & 9 & 5 & 128 \\
\hline
\end{tabular}




\section{Instrumentos}

Medición del grado de participación de los padres en la vida escolar. La medición del grado de participación de los padres en la vida escolar se hizo por medio de la versión francesa de la Encuesta de la Relación entre los Padres y la Escuela (PASS) desarrollada por Ringenberg, Funk, Mullen, Wilford y Kramer (2005). En la presente investigación el coeficiente de confiabilidad alfa de Cronbach resultó bueno, pues alcanzó un índice de .722 .

Medición del rendimiento académico de los estudiantes. Se tomó como indicador del rendimiento académico el resultado del examen oficial estandatizado correspondiente al noveno año fundamental, implementado anualmente por el Ministerio de Educación de Haití.

\section{Análisis de datos}

El coeficiente de correlación $r$ de Pearson se utilizó para determinar la relación entre el grado de involucramiento parental y el rendimiento escolar de sus hijos.

\section{Resultados \\ Análisis descriptivo}

El valor medio de involucramiento parental fue de $86.03(D E=11.26)$, en una escala de valores posibles cuyo rango va de 29 a 145 puntos. El rango observado fue de 49 a 109 puntos.

Las medias de las diferentes dimensiones de involucramiento parental nos permiten ver que los padres están más involucrados en la dimensión paternidad $(M=15,10, D E=3,353)$, seguida de cerca por la de toma de decisiones $(M=15,09, D E=2,817)$, comunicación $(M=14.98 ; D E=$ $2.848)$ y aprendizaje en el hogar $(M=$ 14.58; $D E=2.915)$. Las dimensiones colaboración con la comunidad $(M=$ $13.48, D E=2,988)$ y voluntariado $(M$ $=12.80, D E=3,244)$ mostraron una media bastante menor en comparación con las otras.

La variable rendimiento académico, con un rango posible de 0 a 1800 puntos mostró una media de $1120.54(D E=$ 124.68). Estos datos, junto con los estadísticos descriptivos de los rendimientos por materia, pueden observarse en la Tabla 2.

\section{Análisis correlacional}

El coeficiente de correlación $r$ de Pearson muestra que el rendimiento académico de los estudiantes se relaciona positivamente con la participación de los padres $(r=.299, p=.001)$.

\section{Tabla 2}

Estadísticos descriptivos de rendimiento académico por materia $(N=128)$

\begin{tabular}{lccc}
\hline \multicolumn{1}{c}{ Materia } & Mínimo & Máximo & \multicolumn{1}{c}{$M$} \\
\hline Rendimiento académico general & 747.5 & 1460.0 & 1120.54 \\
Criollo & 138.0 & 285.0 & 228.98 \\
Francés & 100.0 & 256.0 & 184.90 \\
Ciencias Sociales & 111.0 & 291.0 & 222.30 \\
Ciencias Experimentales & 60.0 & 294.0 & 173.34 \\
Matemáticas & 50.0 & 240.0 & 136.67 \\
Lenguaje & 60.0 & 295.5 & 174.34 \\
\hline
\end{tabular}


Dado que el coeficiente es positivo, cuanto mayor sea el involucramiento de los padres en la vida escolar de sus hijos, tanto mayor será el rendimiento académico de estos, aunque debe tenerse en cuenta que el efecto de la relación entre estas dos variables es moderada.

\section{Otros hallazgos}

Se hallaron correlaciones positivas entre el grado de involucramiento parental y el rendimiento en varias materias escolares, evaluadas con el examen oficial estandarizado. Se observó una correlación significativa positiva de involucramiento parental con el rendimiento en Ciencias Sociales $(r=.224, p$ $=.011)$, Lenguaje $(r=.186, p=.035) \mathrm{y}$ Francés $(r=.181, p=.040)$.

También se observó una correlación positiva entre el rendimiento académico y las diferentes dimensiones de la participación de los padres (ver Tabla 3). La mayoría de las dimensiones de la participación de los padres, formuladas por Epstein y Sanders (2006), son predictoras del rendimiento académico. El aprendizaje en el hogar es el mejor indicador del rendimiento académico en el noveno grado $(r=.324, p=.000)$, seguido de la toma de decisiones $(r=$ $.263, p=.003)$, la paternidad $(r=.214$, $p=.015)$ y el voluntariado $(r=.188, p$ $=.034$ ).

En síntesis, se concluye que hay una relación positiva significativa entre el grado de involucramiento de los padres y el rendimiento académico del grupo participantes del estudio.

Entre otros, el estudio mostró los siguientes hallazgos:

1. Los padres están involucrados en diferentes niveles en la vida escolar de sus hijos. La implicación más fuerte se observó en la dimensión paternidad, se- guida de cerca por las dimensiones toma de decisiones, comunicación y aprendizaje en el hogar.

2. La dimensión de la participación de los padres que tiene la mayor correlación con el rendimiento académico es el aprendizaje en el hogar.

3 . En cuanto al rendimiento académico, los estudiantes, en promedio, se desempeñan mejor en Criollo, Ciencias Sociales y Francés. El rendimiento más bajo observado fue en Matemáticas.

4. La correlación del involucramiento parental es mayor con el rendimiento en Ciencias Sociales, Lenguaje y Francés. La correlación más baja se observó con el rendimiento en Criollo.

\section{Tabla 3}

Correlación entre las dimensiones de participación de los padres y rendimiento académico $(\mathrm{N}=128)$

\begin{tabular}{lll}
\hline \multicolumn{1}{c}{ Dimensión } & $r$ & $p$ \\
\hline Paternidad & $.214^{*}$ & .015 \\
Comunicación & .058 & .513 \\
Voluntariado & $.188^{*}$ & .034 \\
Aprendizaje en el hogar & $.324 * *$ & .000 \\
Toma de decisiones & $.263 * *$ & .003 \\
Colaboración & .062 & .490 \\
\hline$* p<.05 . * * p<.01$. & &
\end{tabular}

\section{Discusión}

El análisis de los resultados reveló que existe una correlación positiva y estadísticamente significativa $(r=.299, p<$ .001) entre el grado de involucramiento parental y el rendimiento académico de los hijos. La mejor dimensión del involucramiento parental predictora del rendimiento académico fue el aprendizaje en el hogar $(r=.324, p<.001)$, seguida de la toma de decisiones $(r=.263, p<.001)$. Este resultado muestra que cuanto más involucrados estén los padres en el seguimiento del trabajo en el hogar, tanto más 
elevado será el rendimiento académico de los estudiantes. Los resultados de este trabajo coinciden con los obtenidos por Deslandes y Bertrand (2004, 2005), Castro et al. (2015), Chowa, Ansong y Osei-Akoto (2012) y Viray (2016), quienes observaron una relación positiva entre la participación de los padres en el aprendizaje en el hogar y el rendimiento académico de los estudiantes.

Los resultados del presente estudio muestran una correlación positiva y significativa entre las dos variables, aunque sigue siendo débil, mientras que la mayoría de los estudios (Castro et al., 2015; Doctoroff y Arnold, 2017; Hill y Tyson, 2009; Man Wong et al., 2018) encontraron una fuerte correlación positiva entre el involucramiento parental y el rendimiento académico. Esto se explica por el hecho de que gran parte de la literatura académica se centra en el rendimiento académico en el nivel primario. Sin embargo, Epstein y Dauber (1991) observaron que, a medida que los niños progresan en la escuela secundaria, los padres tienden a involucrarse menos en su vida escolar. De acuerdo con Goodall (2016), los padres creen que no son capaces de acompañarlos en el estudio de temas cada vez más complejos. Además, los niños se vuelven más independientes (Hill y Tyson, 2009). Por lo tanto, es normal que con adolescentes del noveno grado, la correlación sea positiva, pero débil. Aunque diferentes de la tendencia general, estos resultados están muy alineados con los del trabajo realizado por Çiftçi y Nedim Bal (2015).

En este estudio, el análisis de los datos del examen oficial permite observar el rendimiento académico promedio de los estudiantes en cada materia de la prueba. Es interesante notar que los estudiantes en promedio se destacan mejor en Criollo $(M=228.984, D E=27.5646)$, en Ciencias Sociales $(M=222.30, D E=$ 32.1826) y débilmente en Matemáticas $(M=136.67, D E=41.873)$. Sin embargo, de todas las materias, Ciencias Sociales tiene la más alta correlación $(r=$ $.224, p=.011)$ con el involucramiento parental. ¿Es normal que las Ciencias Sociales estén en el primer rango y las matemáticas en la parte inferior de la clasificación de materias correlacionadas con el incolucramiento de los padres? La lógica es clara y comprensible. El material más fácil de seguir por los padres corresponde a las Ciencias Sociales, mientras que las matemáticas constituyen el material más difícil debido a su complejidad.

Hay muchas investigaciones (Fan y Chen, 2001; Henderson y Mapp, 2002; Hill y Tyson, 2009; Jeynes, 2003, 2005, 2007) que confirman que el rendimiento académico de los estudiantes es mejor cuando los padres están involucrados en sus vidas escolares. Esta investigación confirma una vez más la correlación estadísticamente positiva entre el rendimiento académico y la participación de los padres. En consecuencia, los administradores escolares y los padres deben ser lo suficientemente sabios como para recordar que hoy más que nunca deben aunar sus esfuerzos para apoyar mejor a los niños y combatir el fracaso escolar.

\section{Referencias}

Alghazo, R. y Alghazo, Y. (2015). The effect of parental involvement and socioeconomic status on elementary students' mathematics achievement. Journal of Social Sciences and Humanities, 1(5), 521-527.

Alhosani, A. A., Singh, S. K. y Al Nahyan, M.T. (2017). Role of school leadership and climate in student achievement: The mediating role of parental involvement. International Journal of Educational Management, 31(6), 843-851. https://doi.org/10.1108/IJEM-05-2016-0113 


\section{MILORME}

Banco Mundial. (2009). Le système éducatif centrafricain: Contraintes et marges de manœuvre pour la reconstruction du système éducatif dans la perspective de la réduction de la pauvreté. Washington: Autor. https://doi .org/10.1596/978-0-8213-7526-6

Benner, A. D., Boyle, A. E. y Sadler, S. (2016). Parental involvement and adolescents' educational success: The roles of prior achievement and socioeconomic status. Journal of Youth and Adolescence, 45(6), 1-12. https://doi .org/10.1007/s10964-016-0431-4

Bergeron, L., Rousseau, N. y Leclerc, M. (2011). La pédagogie universelle: au cœur de la planification de l'inclusion scolaire. Journal Education et Francophonie, 39(2), 87-104. https:// doi.org/10.7202/1007729ar

Blain, R. (1992). Nouvelles approches pédagogiques: Tous les enfants peuvent réussir. Québec Français, 86, 56-57. Recuperado de https:// id.erudit.org/iderudit/44825ac

Borst, G. y Cachia, A. (2016). Les méthodes en psychologie. París: Presses Universitaires de France.

Castro, M., Expósito-Casas, E., López-Martín, E., Lizasoain, L., Navarro-Asencio, E. y Gaviria, J. (2015). Parental involvement on student academic achievement: A meta-analysis. Educational Research Review, 14, 33-46. https://doi .org/10.1016/j.edurev.2015.01.002

Chowa, G., Ansong, D. y Osei-Akoto, I. (2012). Parental involvement and academic performance in Ghana. Youth Save Research Brief, 42(12). https://doi.org/10.7936/ K74T6HVF

Çiftçi, M. y Nedim Bal, P. (2015). The investigation of the relationship between parents' involvement level and academic achievement on secondary school students. Journal of Human Sciences, 12(1), 363-384. https://doi .org/10.14687/ijhs.v12i1.2974

Deslandes, R. y Bertrand, R. (2004). Motivation des parents à participer au suivi scolaire de leur enfant au primaire. Revue des Sciences de l'Éducation, 30(2), 411-433. https://doi .org/10.7202/012675ar

Deslandes, R. y Bertrand, R. (2005). Motivation of parent involvement in secondary-level schooling. Journal of Educational Research, 98(3), 164-175. https://doi.org/10.3200/ JCER.98.3.164-175

Doctoroff, G. L. y Arnold, D. H. (2017). Doing homework together: The relation between parenting strategies, child engagement, and achievement. Journal of Applied Developmental Psychology, 48, 103-113. https://doi.org/ 10.1016/j.appdev.2017.01.001

Doucet, J. J., Utzschneider, A. y Bourque, J. (2009). Influence parentale sur le rendement scolaire: Comparaison entre des élèves nés au Canada et des élèves nés à l'extérieur du pays. Nouveaux Cahiers de la Recherche en Éducation, 12(2), 227-242. https://doi.org/ 10.7202/1017469ar

Duval, J., Dumoulin, C. y Perron, M. (2014). Collaboration école-famille et prévention du décrochage scolaire: des pistes d'action pour les enseignants du primaire. Revue Canadienne de l'Éducation, 37(3), 1-23.

Epstein, J. L. y Dauber, S. L. (1991). School programs and teacher practices of parent involvement in inner-city elementary and middle schools. Elementary School Journal, 91(3), 289-305. https://doi.org/10.1086/461656

Epstein, J. L. y Sanders, M. G. (2006). Prospects for change: Preparing educators for school, family, and community partnerships. Peabody Journal of Education, 81(2), 81-120. https:// doi.org/10.1207/515327930pje8102_5

Fan, X. y Chen, M. (2001). Parental involvement and students' academic achievement: A meta-analysis. Educational Psychology Review, 13(1), 1-22. https://doi.org/101023/ A: 1009048817385

Flores de Apodaca, R., Gentling, D. G., Steinhaus, J. K. y Rosenberg, E. A. (2015). Parental involvement as a mediator of academic performance among special education middle school students. School Community Journal, 25(2), 35-54.

Foucault, M. (2017). No child left behind: entre espoir et déception. Administration et Éducation, 155(3), 29-33.

Goodall, J. (2016). Technology and school home communication. International Journal of Pedagogies and Learning, 11, 118-131. https:// doi.org/10.1080/22040552.2016.1227252

Grolnick, W. S. (2016). Parental involvement and children's academic motivation and achievement. En W. C. Liu, J. C, Keng Wang y M. Ryan (Eds.), Building autonomous learners: Perspectives from research and practice using self-determination theory (pp. 169-183). Singapore: Springer. https://doi.org/10.1007/978981-287-630-0 9

Henderson, A. T. y Mapp, K. L. (2002). A new wave of evidence: The impact of school, family, and community connections on student achievement. Austin: SEDL.

Hill, N. E. y Tyson, D. F. (2009). Parental involvement in middle school: A meta analytic assessment of the strategies that promote achievement. Developmental Psychology, 45(3), 740-763. https://doi.org/10.1037/a0015362

Jeynes, W. H. (2003). A meta-analysis: The effects of parental involvement on minority children's academic achievement. Education and $U r$ - 


\section{EFECTOS DEL INVOLUCRAMIENTO PARENTAL}

ban Society, 35(2), 202-218. https:// doi .org/10.1177/0013124502239392

Jeynes, W. H. (2005). A meta-analysis of the relation of parental involvement to urban elementary school student academic achievement. Urban Education, 40(3), 237-269. https://doi .org/10. 1177/F0042085905274540

Jeynes, W. H. (2007). The relationship between parental involvement and urban secondary school student academic achievement: A meta-analysis. Urban Education, 42(1), 82-110. https:// doi.org/10.1177F0042085906293818

Man Wong, R. S., Wing Ho, F. K., San Wong, W. H., Suen Tung, K. T., Bong Chow, C., Rao, N., Ling Chang, K. e Ip, P. (2018). Parental involvement in primary school education: Its relationship with children's academic performance and psychosocial competence through engaging children with school. Journal of Child and Family Studies, 27, 1544-1555. https://doi .org/10.1007/s10826-017-1011-2

Mestari, S. (2001). Les classes relais: travailler en réseau pour prévenir l'exclusion scolaire. Recuperado de https://documentation.ehesp.fr/ memoires/2001/men/mestari.pdf

Ministère de 1'Éducation Nationale et de la Formation Professionnel (2012). Port-au-Prince. Vers la refondation du système éducatif haïtien: plan opérationnel 2010-2015 des recommandations du groupe de travail sur l'éducation et la formation. Recuperado de http://planipolis .iiep.unesco.org/sites/planipolis/files/ressources /haiti_plan_operationnel_2010-2015.pdf

Nanhou, V., Desrosiers, H. et Belleau, L. (2013). La collaboration parent-école au primaire: le point de vue des parents. Institut de la statistique du
Québec, 7(3). Recuperado de http://www.stat .gouv.qc.ca/statistiques/education/prescolaireprimaire/collaboration-parent-ecole.html

Oyerinde, B. A. (2014). Correlating parental involvement and mathematics achievement of African American eighth-grade students (Publicación No. 3620303) [Tesis doctoral]. ProQuest Dissertations and Theses Global.

Ringenberg, M. C., Funk, V., Mullen, K., Wilford, A. y Kramer, J. (2005). The test-retest reliability of the Parent and School Survey (PASS). School Community Journal, 15(2), 121-134.

Ross, T. (2016). The differential effects of parental involvement on high school completion and postsecondary attendance. Education Policy Analysis Archives, 24(30), 1-38. https://doi .org/10.14507/epaa.v24.2030

Thomas, V., De Backer, F., Peeters, J. y Lombaerts, K. (2019). Parental involvement and adolescent school achievement: The mediational role of self-regulated learning. Learning Environment Research, 22(3), 345-363. https://doi .org/10.1007/s10984-019-09278-x

Viray, J. S. (2016). Parental involvement as predictor of student academic performance. Imperial Journal of Interdisciplinary Research, 2(6), 1379-1382.

Wilder, S. (2014). Effects of parental involvement on academic achievement: A meta-synthesis. Educational Review, 66(3), 377-397. https:// doi.org/10.1080/00131911.2013.780009

Recibido: 4 de febrero de 2019

Revisado: 25 de marzo de 2019 Aceptado: 8 de abril de 2019 